\title{
MICU1 occludes MCU in the mitochondrial calcium uniporter complex
}

\author{
Chen-Wei Tsai ${ }^{1}$, Anna Van Keuren ${ }^{1}$, John Bankston $^{1}$, Zhiwei $\mathrm{Ma}^{2}$, Ming-Feng Tsai ${ }^{1,3}$
}

1. Department of Physiology and Biophysics, University of Colorado Anschutz Medical Campus, Aurora, CO 80045.

2. Dalton Cardiovascular Research Center, University of Missouri, Columbia, MO 65211.

3. Correspondence should be addressed to Ming-Feng Tsai (ming-feng.tsai@cuanschtuz.edu)

\begin{abstract}
The mitochondrial calcium uniporter imports cytoplasmic $\mathrm{Ca}^{2+}$ into the mitochondrial matrix to regulate cell bioenergetics, $\mathrm{Ca}^{2+}$ signaling, and apoptosis. The uniporter contains the pore-forming MCU subunit, an EMRE protein that binds to MCU, and the regulatory MICU1/MICU2 subunits. Structural and biochemical studies have suggested that MICU1 gates MCU by blocking and unblocking the $\mathrm{Ca}^{2+}$ pore. However, mitoplast patch-clamp experiments argue that MICU1 does not block $\mathrm{Ca}^{2+}$ transport but instead potentiates MCU. To address this direct clash of proposed MICU1 function, we applied purified MICU1 to $\mathrm{Ca}^{2+}$-conducting MCU-EMRE subcomplexes in outside-out patches excised from Xenopus oocytes. MICU1 strongly inhibits $\mathrm{Ca}^{2+}$ currents, and the inhibition is abolished by mutating an MCU-interacting K126 residue in MICU1. Further experiments show that MICU1 block was not observed in mitoplasts because MICU1 dissociates from the uniporter complex. These results firmly establish that MICU1 shuts the uniporter in resting cellular conditions.
\end{abstract}

\section{Introduction}

The mitochondrial $\mathrm{Ca}^{2+}$ uniporter is a multi-subunit $\mathrm{Ca}^{2+}$ channel complex in the inner mitochondrial membrane (IMM). Its transmembrane (TM) region contains the MCU subunit, which forms a tetrameric $\mathrm{Ca}^{2+}$ pore $^{1-3}$, and the EMRE protein that binds to MCU to stabilize the pore's conducting conformation ${ }^{4,5}$. The MICU subunits, which include MICU1, MICU2, and the neuron-specific MICU3 (not discussed here), contain two canonical EF-hands and dwell in the intermembrane space (IMS) to regulate uniporter activity $^{1-3,6}$. MICU1 regulation of the uniporter is important for normal physiology, as MICU1 mutations have been linked to severe neuromuscular disorders in humans ${ }^{7,8}$. The exact regulatory function of MICU1, however, is currently under debate.

Biochemical and functional analyses ${ }^{1-3,9,10}$ have suggested a mechanism that MICU1 (but not MICU2) binds to the $\mathrm{Ca}^{2+}$-coordinating Asp ring (D261 in human MCU) at the cytoplasmic entrance of the MCU pore to block $\mathrm{Ca}^{2+}$ flux at resting levels of cytoplasmic $\mathrm{Ca}^{2+}(100-200 \mathrm{nM})$. Upon elevation of local $\mathrm{Ca}^{2+}$, MICU1 dissociates from the pore to enable $\mathrm{Ca}^{2+}$ permeation (Fig. 1A). This "occlusion model" is supported by multiple key results: first, co-immunoprecipitation shows that human MCU (hMCU) can complex with MICU1 in the absence of EMRE, and that such MCU-MICU1 interaction is disrupted by a D261A mutation in $\mathrm{hMCU}^{9}$; second, a quantitative ${ }^{45} \mathrm{Ca}^{2+}$ flux assay shows that substituting wild-type (WT) hMCU with the D261A mutant in WT HEK293 cells leads to robust mitochondrial $\mathrm{Ca}^{2+}$ uptake at resting $\mathrm{Ca}^{2+}$, phenocopying MICU1-knock out $(\mathrm{KO})^{9}$; and third, MICU1 suppresses inhibition of MCU by Ru360, which binds to D261 ${ }^{10}$. Recent cryo-electron microscope (cryo-EM) studies appear to provide the structural basis of the occlusion model ${ }^{1-3}$. In low $\mathrm{Ca}^{2+}$, MICU1 uses a 5-residue Arg/Lys ring to surround 
MCU's D261-ring to block the channel (Fig. 1B). $\mathrm{Ca}^{2+}$ binding to MICU1 induces conformational changes that disrupt MICU1-MCU interactions to open the pore (Fig. 1B). Functional work ${ }^{3}$ further shows that mutations in the Arg/Lys ring abolish MICU1 block of uniporter $\mathrm{Ca}^{2+}$ transport in resting cellular $\mathrm{Ca}^{2+}$.

The occlusion model, however, directly clashes with results in a landmark study, which employs patchclamp of mitoplast (a submitochondrial vesicle rid of the outer membrane) to demonstrate that the uniporter is a bona fide $\mathrm{Ca}^{2+}$ channel ${ }^{11}$. In this work, it was shown that the uniporter can conduct $\mathrm{Na}^{+}$in 0 $\mathrm{Ca}^{2+}$, apparently contradicting the idea that MICU1 physically occludes the MCU pore under such a 0 $\mathrm{Ca}^{2+}$ condition. Expanding on these observations, a recent electrophysiological work ${ }^{12}$ further proposes a "potentiation model" for MICU1 (Fig. 1C). Based on the finding that MICU1-KO reduces uniporter $\mathrm{Ca}^{2+}$ currents by $\sim 50 \%$ in mitoplasts, it was argued that MICU1's function is to potentiate the uniporter rather than to block MCU. The goal of this work is to understand why rigorous work from multiple labs over the last two decades produce two entirely opposite models underlying $\mathrm{Ca}^{2+}$-dependent gating of the uniporter by MICU1.

\section{Results}

\section{Purified, EF-hand mutated MICU1 blocks MCU $\mathrm{Ca}^{2+}$ currents}

The critical difference between the occlusion and potentiation models is that the former demands the presence of a MICU1-mediated, blocked state, while the latter argues that MICU1-block is nonexistent. Thus, the key to determine which of these two models is correct is to examine if there is a blocked state. We thus test if purified MICU1 can inhibit MCU currents, analogous to classical $\mathrm{K}^{+}$-channel work ${ }^{13,14}$ showing that purified neurotoxins (e.g. charybdotoxin) or synthesized ball peptides (as in the ball-andchain mechanism) block heterologously expressed $\mathrm{K}^{+}$channels. Such a strategy was deemed feasible as cryo-EM work has already shown that purified MICU1 can bind to the MCU-EMRE pore subcomplex reconstituted in lipid bilayers ${ }^{1}$. We excised outside-out patches from Xenopus oocytes expressing a human MCU-EMRE (hME) fusion protein in the cellular membrane. Our previous work ${ }^{15}$ demonstrated that hME exhibits similar electrical properties as the uniporter recorded in mitoplast patch-clamp ${ }^{11}$. At negative voltages, hME conducts inward $\mathrm{Ca}^{2+}$ currents that are strongly and reversibly inhibited by Ru360 (Fig. 2A). We then perfused MICU1 $1_{\mathrm{EF}}$, which includes 4 mutations (D231A, E242K, D421A, E432K) to eliminate $\mathrm{Ca}^{2+}$ binding to the EF hands to mimic low $\mathrm{Ca}^{2+}$ conditions. It has been shown qualitatively that transiently expressed MICU1 $1_{\mathrm{EF}}$ in $\mathrm{HEK}$ cells suppresses mitochondrial $\mathrm{Ca}^{2+}$ uptake ${ }^{16}$, a result recapitulated here using quantitative ${ }^{45} \mathrm{Ca}^{2+}$ flux (Fig. 2B). Strikingly, applying MICU1 $1_{\mathrm{EF}}$ reduces macroscopic hME $\mathrm{Ca}^{2+}$ currents by $86 \pm 7 \%$, with a current decay time constant of $44 \pm 12 \mathrm{~s}$ (i-ii, Fig. 2A). Moreover, in recordings wherein single-channel transitions can be observed (iii-iv, Fig. 2A), MICU1 $1_{\mathrm{EF}}$ greatly reduces $\mathrm{hME}$ open time and open probability. The inhibition is not reversible with a $3-$ $5 \mathrm{~min}$ wash. These results are fully explained by a molecular picture that purified MICU1 $1_{\mathrm{EF}}$ binds to hME with a slow on-rate to block hME in a highly stable MICU1 $1_{\mathrm{EF}}-\mathrm{hME}$ complex (scheme I).

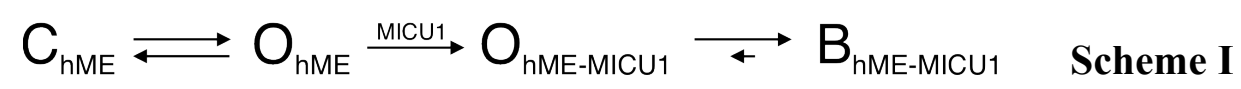

To test if MICU1 $1_{\mathrm{EF}}$ inhibits hME via the blockade interactions observed in uniporter-complex structures (Fig. 1B), we mutated MICU1's K126 residue, which contacts MCU's D261 ring to block the pore. ${ }^{45} \mathrm{Ca}^{2+}$ flux shows that K126E abolishes the ability of transiently expressed MICU1 $1_{\mathrm{EF}}$ to inhibit mitochondrial $\mathrm{Ca}^{2+}$ uptake in HEK cells (Fig. 2B). Moreover, perfusing K126E-MICU1 $1_{\mathrm{EF}}$ leaves macroscopic hME Ca ${ }^{2+}$ currents unchanged, indicating that K126E indeed abolishes MICU1 $1_{\mathrm{EF}}$ block (Fig. 2C). Size-exclusion 
chromatography shows that purified K126E-MICU1 $1_{\mathrm{EF}}$ preserves the proper biochemical behavior of MICU1 $1_{\mathrm{EF}}$ (Fig. 2D), suggesting that K126E does not perturb MICU1 folding. Taken together, our electrical recordings demonstrate that $\mathrm{MICU} 1_{\mathrm{EF}}$ directly inhibits $\mathrm{MCU}$ via a K126-mediated interaction observed in the blocked state of the uniporter-complex structure in $0 \mathrm{Ca}^{2+}$ (Fig. 1A-B). These results thus present novel electrophysiological evidences that support the occlusion mechanism and cannot be explained by the potentiation model.

\section{No MICU1 block of MCU in mitoplasts because MICU1 is dissociated}

We then sought to understand why MICU1 block was not observed in mitoplasts ${ }^{11}$. As MICU1 contacts the uniporter's TM subunits via electrostatic interactions ${ }^{3,9}$, it is possible that high-salt treatments in the mitoplast production procedure might lead to MICU1 dissociation from the TM subunits ${ }^{11}$. Accordingly, we employed quantitative Western blots to compare the MICU1/MCU signal ratio in mitochondria vs mitoplasts produced following published procedures in the original mitoplast patch-clamp experiments ${ }^{11}$. Results show a 30\% reduction of MICU1/MCU ratio in mitoplasts (Fig. 3A), suggesting that $\sim 30 \%$ of MICU1 dissociates into the bulk solution during mitoplast formation.

Since the uniporter preferentially dwells in areas near the inner/outer membrane contact sites $^{3}$, the disruption of such contact sites in mitoplasts could effectively dilute the uniporter in a much bigger surface area, rendering it more difficult for dissociated MICU1 to bind back to the TM region. We thus wondered if a portion of remaining MICU1 in mitoplasts might only attach to the IMM without being associated with the uniporter (MICU1 is a peripheral membrane protein). To this end, the uniporter complex was analyzed using blue native PAGE (Fig. 3B). The uniporter in WT mitochondria runs as a smear (lane 3), likely due to the presence of uniporter complexes binding different numbers of MICU1. Accordingly, the smear is eliminated by MICU1-KO (lanes 5-6). MICU1-KO reduces EMRE expression $^{17}$, thus creating EMRE-bound (upped band, lanes 5-6) and EMRE-free (lower band) complexes. The latter is also observed in EMRE-KO samples (lane 1). In WT mitoplasts, the uniporter runs as a single band without a smear (lane 4), and the band position is consistent with the EMRE-bound, MICU1-free uniporter (lane 5-6). These results thus argue strongly that the mitoplast production process leads to dissociation of MICU1 from the uniporter complex (Fig. 3C), creating a subpopulation of MICU1-free uniports that can conduct $\mathrm{Na}^{+}$currents in $0 \mathrm{Ca}^{2+}$.

To further test the scheme in Fig. 3C using functional assays, we analyzed ${ }^{45} \mathrm{Ca}^{2+}$ flux into mitochondria or mitoplasts. In $250 \mathrm{nM} \mathrm{Ca}^{2+}$, mitochondria from MICU1-KO HEK cells take up much more $\mathrm{Ca}^{2+}$ than WT mitochondria (Fig. 3D), as expected from the loss of MICU1 block in the occlusion model (Fig. 1A). However, WT mitoplasts take up comparable amount of $\mathrm{Ca}^{2+}$ in $250 \mathrm{nM} \mathrm{Ca}^{2+}$ as MICU1-free mitoplasts (Fig. 3D), thus demonstrating that MICU1 regulation of the uniporter is severely perturbed in mitoplasts.

\section{Discussion}

The uniporter complex emerges in eukaryotic evolution with only MCU and MICU1 subunits ${ }^{18}$, suggesting that MICU1 must regulate MCU through direct physical contacts. Indeed, the MCU-MICU1 interaction is so conserved that human MICU1 can complex with MCU homologues in A. thaliana (plant), D. discoideum (amoeba) ${ }^{9}$, or T. castaneum (beetle) ${ }^{2}$. When MCU structures were first determined, its small and flat IMS surface makes it rather obvious that docking of MICU1 onto this surface would block ion permeation through the $\mathrm{Ca}^{2+}$ pore ${ }^{19}$. This is indeed confirmed by subsequent biochemical and structural work summarized in the Introduction, leading to the establishment of the occlusion mechanism (Fig. 1A). In the potentiation model, perhaps realizing that MICU1 would unlikely increase uniporter 
activity by binding to MCU's IMS surface, it was proposed that MICU1 potentiates MCU allosterically through EMRE (Fig. 1C) ${ }^{12}$. Such an argument, however, lacks apparent structural basis, as EMRE-MCU interactions and the MCU pore conformation in the MCU-EMRE-MICUs structure appear identical in low and high $\mathrm{Ca}^{2+}$ conditions $^{1-3}$. Moreover, EMRE is only present in animals - an EMRE-dependent potentiation mechanism would imply that the highly conserved MICU1 protein, which co-evolves with MCU since early eukaryotes ${ }^{18}$, actually has no function in the uniporter in non-metazoan organisms.

Our electrical recordings, coupled with structural information, directly demonstrate MICU1 block of the MCU pore in real time, a phenomenon that the potentiation model cannot explain. Moreover, our biochemical and functional investigations resolve the longstanding puzzle about why the uniporter can conduct $\mathrm{Na}^{+}$without $\mathrm{Ca}^{2+}$ in mitoplast membranes. However, we note that it remains unclear why MICU1-KO reduces uniporter $\mathrm{Ca}^{2+}$ currents in mitoplast patch-clamp experiments ${ }^{12}$. Our work (under consideration elsewhere) shows that MICU1-KO indeed reduces uniporter-mediated ${ }^{45} \mathrm{Ca}^{2+}$ uptake, but such reduction is caused by EMRE downregulation (rather than loss of MICU1 potentiation), because transient EMRE expression in MICU1-KO HEK cells restores uniporter activity to WT-cell levels. However, mitoplast patch-clamp shows that overexpressing EMRE does not further enhance macroscopic $\mathrm{Ca}^{2+}$ currents in MICU1-free mitoplasts ${ }^{12}$. We do not know how to explain the discrepancy, but would like to point out that proper expression of EMRE in MICU1-KO cells must be carefully confirmed with quantitative methods, given EMRE expression is strongly suppressed in these cells.

\section{Materials and Methods}

\section{Electrophysiology}

Xenopus oocytes were injected with $60 \mathrm{ng}$ of cRNA encoding the human MCU-EMRE fusion protein (hME) and were incubated in an ND96 solution $(2 \mathrm{mM} \mathrm{KCl}, 96 \mathrm{mM} \mathrm{NaCl}, 1.8 \mathrm{mM} \mathrm{CaCl}, 1 \mathrm{mM} \mathrm{MgCl}$, $5 \mathrm{mM}$ HEPES, $\mathrm{pH}$ 7.4-NaOH) at $17^{\circ} \mathrm{C}$ as described in our previous work ${ }^{15}$. Patch-clamp recordings were performed 3-5 days after cRNA injection. Borosilicate pipettes were produced using a Sutter P1000 pipette puller, and were fire polished with a homemade microforge, leading to a pipette resistance of $3-5$ $\mathrm{M} \Omega$. In all experiments, the pipet solution contains $90 \mathrm{mM}$ sodium gluconate, $10 \mathrm{mM} \mathrm{NaCl}, 5 \mathrm{mM}$ EGTA, 20 HEPES, pH 7.6-NaOH. Outside-out patches were excised from oocytes in a continuous perfusion ND96 bath, and then moved to the outlet of a fast solution change system (Warner SF-77B) to be perfused with a Ca-100 solution (100 $\mathrm{mM} \mathrm{CaCl}_{2}, 20 \mathrm{mM}$ HEPES, $\mathrm{pH}$ 7.6-NaOH). Currents of hME were recorded at room temperature with a HEKA EPC-10 amplifier, filtered at $100 \mathrm{~Hz}$ with an eight-pole Bessel filter (Warner), and digitized at a frequency of $500 \mathrm{~Hz}$. The membrane potential was clamped at 60 to $-100 \mathrm{mV}$ (more negative voltages were used when the seal was more stable). Negative (inward) currents are defined as currents from the bath to the cytoplasmic side of outside-out membranes. $100 \mathrm{nM}$ Ru360 or $10 \mu \mathrm{M}$ MICU1 were diluted in Ca-100 and was applied to the patches via the SF-77B fast solution change system.

\section{Protein purification}

DNA encoding N-terminally maltose binding protein (MBP)-tagged MICU1 proteins was cloned into a pET21b vector. Transformed $E$. coli BL21 cells were inoculated into terrific broth, grown to an OD of 1.0 at $37^{\circ} \mathrm{C}$, and then induced for protein expression by adding $0.1 \mathrm{mM}$ IPTG for $2 \mathrm{~h}$ at $37^{\circ} \mathrm{C}$. Cells were pelleted, resuspended in a breaking buffer (BB, $500 \mathrm{mM} \mathrm{NaCl}, 25 \mathrm{mM}$ HEPES, pH 7.5-NaOH) supplemented with $1 \mathrm{mg} / \mathrm{mL}$ leupeptin/pepstatin and $1 \mathrm{mM}$ PMSF. After lysis by sonication, the lysate is clarified by centrifugation at $16,000 \mathrm{~g}$ for $30 \mathrm{~min}$ at $4{ }^{\circ} \mathrm{C}$. The lysate wan then loaded onto a cobalt- 
affinity column. The column was washed and eluted with $10 \mathrm{mM}$ and $200 \mathrm{mM}$ imidazole in BB, respectively. The protein was then concentrated and further purified via a Superdex 200 Increase sizeexclusion column equilibrated with BB. Elution volume $10.5-12 \mathrm{~mL}$ was collected and concentrated down to $150 \mu \mathrm{M}$. The concentrated stock was stored at $-80^{\circ} \mathrm{C}$ and diluted to $\mathrm{Ca}-100$ right before electrophysiological experiments to be used within $4 \mathrm{hr}$. The freeze-thaw process and leaving protein in Ca-100 for $4 \mathrm{hr}$ in room temp does not affect the size-exclusion profiles. The yield of WT-MICU1, $\mathrm{MICU}_{\mathrm{EF}}$, and K126E-MICU1 $1_{\mathrm{EF}}$ are $15 \mathrm{mg}, 5 \mathrm{mg}, 2.5 \mathrm{mg} / \mathrm{L}$ E.coli.

\section{Cell culture and molecular biology}

HEK $293 \mathrm{~T}$ cells were cultured in DMEM with $10 \% \mathrm{FBS}$ in a $5 \% \mathrm{CO}_{2}$ incubator at $37{ }^{\circ} \mathrm{C}$. MICU1-KO was achieved by CRISPR-Cas9, and has been validated in our previous publication ${ }^{17}$. The genes encoding MICU1 mutants were cloned into a pcDNA3.1(+) vector for transient expression using Lipofectamine 3000 (Life Technologies). Site-directed mutagenesis was performed using a Quick Change kit (Agilent) with the mutations verified by Sanger sequencing.

\section{Mitochondria/mitoplast production, Western blot, and blue-native (BN)-PAGE}

Mitochondria and mitoplasts were produced following the protocols described in the original mitoplast patch-clamp paper ${ }^{11}$ with some minor modifications. Briefly, HEK cells suspended in a mitochondrial resuspension buffer (250 mM sucrose, $5 \mathrm{mM}$ HEPES, $1 \mathrm{mM}$ EGTA, $\mathrm{pH}$ 7.2-KOH) were disrupted by passing through 27.5 gauge needles $10-20$ times. Mitochondria were pelleted by differential centrifugation (1000 g once followed by $13,000 \mathrm{~g} 3$ times) at $4{ }^{\circ} \mathrm{C}$. To obtain mitoplasts, mitochondria were resuspended in an osmotic shock buffer ( $5 \mathrm{mM}$ sucrose, $5 \mathrm{mM}$ HEPES, $1 \mathrm{mM}$ EGTA, pH 7.2-KOH) for $10 \mathrm{~min}$ on ice, spun down at $13,000 \mathrm{~g}$ for $10 \mathrm{~min}$ at $4{ }^{\circ} \mathrm{C}$, resuspended in a high-salt buffer $(750 \mathrm{mM}$ $\mathrm{KCl}, 100 \mathrm{mM}$ HEPES, $1 \mathrm{mM}$ EGTA, $\mathrm{pH} 7.2-\mathrm{KOH})$ for $30 \mathrm{~min}$ on ice, and then pelleted by centrifugation at $13,000 \mathrm{~g}$ for $10 \mathrm{~min}$ at $4{ }^{\circ} \mathrm{C}$.

To perform Western blots as in Fig. 3A, samples obtained in the mitoplast production process were subjected to SDS-PAGE and transfer to PVDF membranes. The membranes were treated with both antiMICU1 (Sigma, HPA034780, 1:5,000) and anti-MCU (Cell Signaling, D2Z3B, 1:10,000) antibodies at 4 ${ }^{\circ} \mathrm{C}$ for overnight, followed by a IRDye 680 goat anti-rabbit secondary antibody (Li-Cor, 1:10,000) in room temperature for $1 \mathrm{~h}$. The image was acquired using a Li-Cor Odyssey CLx imager.

BN-PAGE was performed using the Novex NativePAGE Bis-Tris gel system (Life Technologies). $200 \mu \mathrm{g}$ of mitochondria or $45 \mu \mathrm{g}$ of mitoplasts was dissolved in $20 \mu \mathrm{L}$ of NativePAGE buffer supplied with $4 \%$ digitonin, $4 \%$ glycerol, and $0.5 \%$ G-250. Samples were loaded onto $4-16 \%$ Bis-Tris gels, subjected to electrophoresis at $4{ }^{\circ} \mathrm{C}$, and transferred to PVDF membranes. The membranes were de-stained by a solution containing $25 \%$ methanol and $10 \%$ acetic acid, blocked with $5 \%$ milk in TBS (140 mM NaCl, 3 $\mathrm{mM} \mathrm{KCl}, 25 \mathrm{mM}$ HEPES, $\mathrm{pH}$ 7.4- $\mathrm{HCl}$ ), and then incubated with anti-MCU antibody (Cell Signaling, $\mathrm{D} 2 \mathrm{Z3B}, 1: 5000$ ) in TBST (TBS $+0.05 \%$ Tween 20 ) for overnight at $4{ }^{\circ} \mathrm{C}$, followed by anti-rabbit alkaline phosphatase-conjugated secondary antibody (Promega, S3731, 1:5,000 in TBST) at room temperature for $1 \mathrm{~h}$. Colorimetric detection was performed by adding NBT/BCIP substrates (Life Technologies) to the membranes.

\section{Mitochondrial and mitoplast ${ }^{45} \mathrm{Ca}^{2+}$ uptake assays}

To measure ${ }^{45} \mathrm{Ca}^{2+}$ flux into mitochondria, mitochondria were isolated using procedures described above and quantified using the BCA assay (Pierce). $350 \mu \mathrm{g}$ of mitochondria were resuspended in $1 \mathrm{~mL}$ of a 
wash buffer (WB, $120 \mathrm{mM} \mathrm{KCl,} 25 \mathrm{mM}$ HEPES, $2 \mathrm{mM} \mathrm{KH}_{2} \mathrm{PO}_{4}, 1 \mathrm{mM} \mathrm{MgCl}$, pH 7.2-KOH), before being pelleted. ${ }^{45} \mathrm{Ca}^{2+}$ uptake was initiated by resuspending mitochondria in $350 \mu \mathrm{L}$ of a low $\mathrm{Ca}^{2+}$ recording buffer (LCRB, $120 \mathrm{mM} \mathrm{KCl}, 25 \mathrm{mM}$ HEPES, $2 \mathrm{mM} \mathrm{KH}_{2} \mathrm{PO}_{4}, 5 \mathrm{mM}$ succinate, $1 \mathrm{mM} \mathrm{MgCl}_{2}$, $0.69 \mathrm{mM}$ EGTA, $0.5 \mathrm{mM} \mathrm{CaCl}, \mathrm{pH} 7.2-\mathrm{KOH}$ ), and was terminated at 2, 4, and 6 min by adding $100 \mu \mathrm{L}$ of the suspension into $5 \mathrm{~mL}$ of ice-cold $\mathrm{WB}$, and filtered through nitrocellulose membranes. The membranes were extracted in a scintillation cocktail for scintillation counting. Th rate of mitochondrial $\mathrm{Ca}^{2+}$ uptake was obtained by linear fit of data points, with detailed calculation methods provided in our previous work ${ }^{9}$. Mitoplast ${ }^{45} \mathrm{Ca}^{2+}$ flux was performed in a similar manner, with the following difference: $\mathrm{Ca}^{2+}$ uptake was quenched by adding $100 \mu \mathrm{L}$ mitoplasts to $1 \mathrm{~mL}$ of ice-cold WB supplemented with 200 $\mathrm{nM}$ Ru360 and $5 \mu \mathrm{M}$ CGP-37157, and external ${ }^{45} \mathrm{Ca}^{2+}$ was removed by spinning down mitoplasts at $13,000 \mathrm{~g}$ for $10 \mathrm{~min}$ at $4{ }^{\circ} \mathrm{C}$. All ${ }^{45} \mathrm{Ca}^{2+}$ uptake experiments were performed in the presence or absence of $200 \mathrm{nM}$ Ru360 to allow isolation of uniporter-specific mitochondrial $\mathrm{Ca}^{2+}$ uptake.

\section{Data analysis and statistics}

Data points were presented as mean \pm S.E.M. Statistical analysis was performed using t-test, with significance defined as $p<0.05$. All experiments were done in at least 3 independent repeats. Analysis of electrophysiological data was done using Igor Pro 8 (WaveMetrics). Western blot quantification was done using the Li-Cor Image Studio software (version 5.2).

\section{Acknowledgements}

We thank Madison Rodriguez and Dr. Han-I Yeh for technical assistance, and Dr. Christopher Miller for critical reading of the manuscript. We thank Dr. Yuriy Kirichok and Dr. Vivek Garg for offering two rounds of in-depth reviews and multiple days of intense but respectful email debates, leading to strongly improved manuscript. We thank Dr. György Hajnóczky for discussions during the development of this work. CT, AV, and MF are supported by the NIH grant R01-GM129345.

\section{Author Contributions}

MT conceptualized the project; CT, AV, and ZM performed research. CT and AV analyzed data. JB supplied key resource. MT wrote the paper.

\section{Declaration of Interests}

The authors declare no competing interests. 


\section{References}

1 Wang, Y. et al. Structural insights into the $\mathrm{Ca}(2+)$-dependent gating of the human mitochondrial calcium uniporter. Elife 9, doi:10.7554/eLife.60513 (2020).

2 Wang, C., Jacewicz, A., Delgado, B. D., Baradaran, R. \& Long, S. B. Structures reveal gatekeeping of the mitochondrial $\mathrm{Ca}(2+)$ uniporter by MICU1-MICU2. Elife 9, doi:10.7554/eLife.59991 (2020).

3 Fan, M. et al. Structure and mechanism of the mitochondrial $\mathrm{Ca}(2+)$ uniporter holocomplex. Nature 582, 129-133, doi:10.1038/s41586-020-2309-6 (2020).

4 Van Keuren, A. M. et al. Mechanisms of EMRE-Dependent MCU Opening in the Mitochondrial Calcium Uniporter Complex. Cell Rep 33, 108486, doi:10.1016/j.celrep.2020.108486 (2020).

5 Sancak, Y. et al. EMRE is an essential component of the mitochondrial calcium uniporter complex. Science 342, 1379-1382, doi:10.1126/science.1242993 (2013).

6 Wang, L. et al. Structural and mechanistic insights into MICU1 regulation of mitochondrial calcium uptake. EMBO J 33, 594-604, doi:10.1002/embj.201386523 (2014).

7 Musa, S. et al. A Middle Eastern Founder Mutation Expands the Genotypic and Phenotypic Spectrum of Mitochondrial MICU1 Deficiency: A Report of 13 Patients. JIMD Rep 43, 79-83, doi:10.1007/8904_2018_107(2019).

8 Logan, C. V. et al. Loss-of-function mutations in MICU1 cause a brain and muscle disorder linked to primary alterations in mitochondrial calcium signaling. Nat Genet 46, 188-193, doi:10.1038/ng.2851 (2014).

9 Phillips, C. B., Tsai, C. W. \& Tsai, M. F. The conserved aspartate ring of MCU mediates MICU1 binding and regulation in the mitochondrial calcium uniporter complex. Elife 8, doi:10.7554/eLife.41112 (2019).

10 Paillard, M. et al. MICU1 Interacts with the D-Ring of the MCU Pore to Control Its Ca(2+) Flux and Sensitivity to Ru360. Mol Cell 72, 778-785 e773, doi:10.1016/j.molcel.2018.09.008 (2018).

11 Kirichok, Y., Krapivinsky, G. \& Clapham, D. E. The mitochondrial calcium uniporter is a highly selective ion channel. Nature 427, 360-364, doi:10.1038/nature02246 (2004).

12 Garg, V. et al. The mechanism of MICU-dependent gating of the mitochondrial $\mathrm{Ca}(2+)$ uniporter. Elife 10, doi:10.7554/eLife.69312 (2021).

13 Park, C. S. \& Miller, C. Interaction of charybdotoxin with permeant ions inside the pore of a K+ channel. Neuron 9, 307-313 (1992). 
14 Zagotta, W. N., Hoshi, T. \& Aldrich, R. W. Restoration of inactivation in mutants of Shaker potassium channels by a peptide derived from ShB. Science 250, 568-571, doi:10.1126/science.2122520 (1990).

15 Tsai, C. W. \& Tsai, M. F. Electrical recordings of the mitochondrial calcium uniporter in Xenopus oocytes. J Gen Physiol 150, 1035-1043, doi:10.1085/jgp.201812015 (2018).

16 Kamer, K. J. \& Mootha, V. K. MICU1 and MICU2 play nonredundant roles in the regulation of the mitochondrial calcium uniporter. EMBO Rep 15, 299-307, doi:10.1002/embr.201337946 (2014).

17 Tsai, C. W. et al. Proteolytic control of the mitochondrial calcium uniporter complex. Proc Natl Acad Sci U S A 114, 4388-4393, doi:10.1073/pnas.1702938114 (2017).

18 Pittis, A. A. et al. Discovery of EMRE in fungi resolves the true evolutionary history of the mitochondrial calcium uniporter. Nat Commun 11, 4031, doi:10.1038/s41467-020-17705-4 (2020).

19 Yoo, J. et al. Cryo-EM structure of a mitochondrial calcium uniporter. Science 361, 506-511, doi:10.1126/science.aar4056 (2018). 


\section{Figure 1}
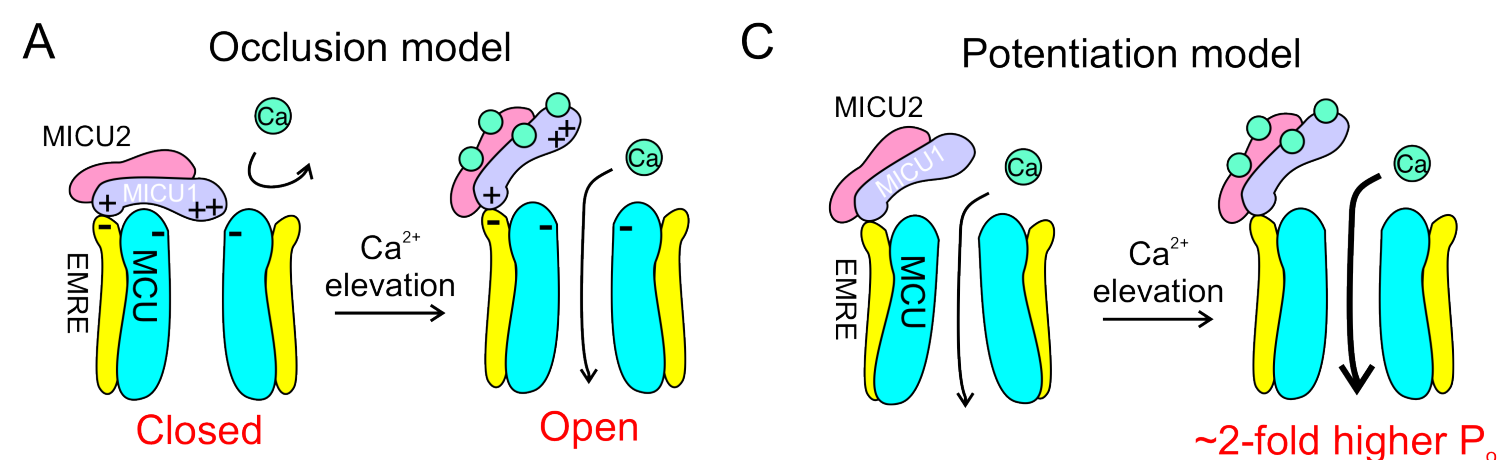

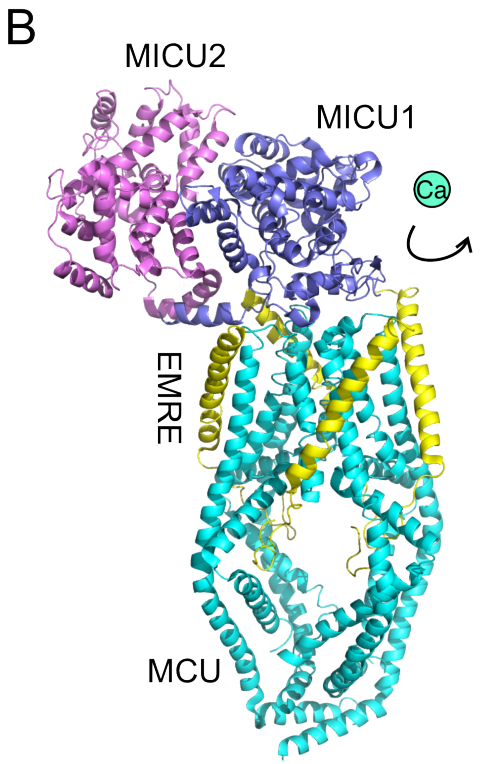

Closed

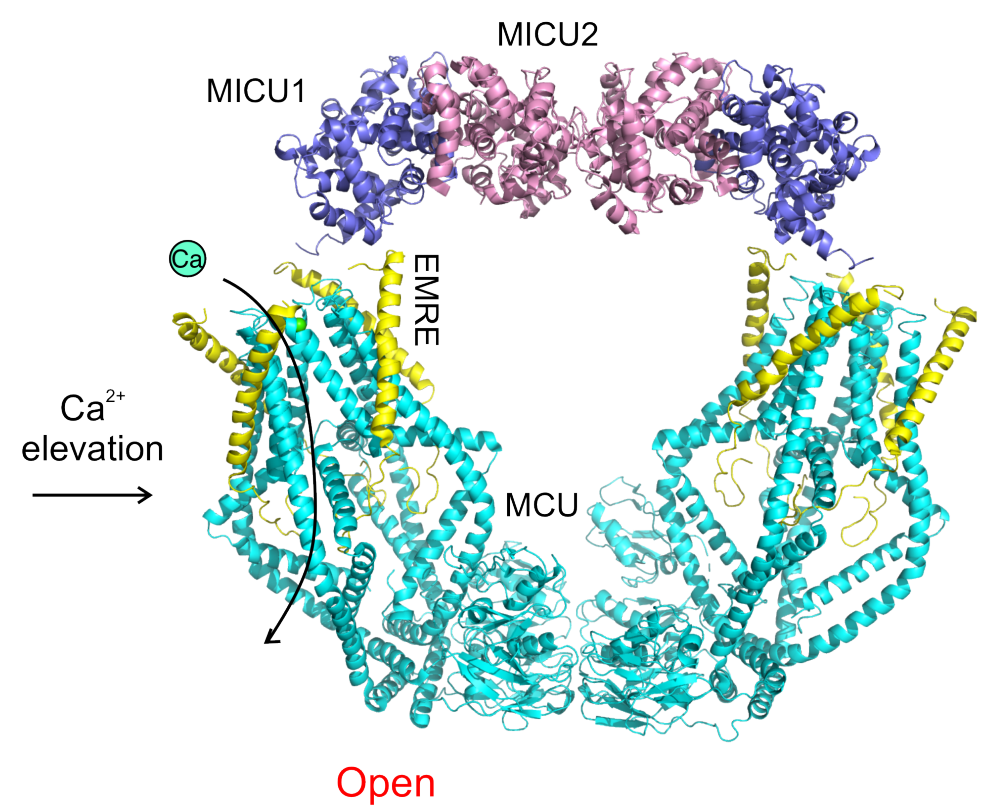

Figure 1. Two models for $\mathrm{Ca}^{2+}$-dependent gating of the uniporter. (A) The occlusion model. The cartoon illustrates that MICU1 interacts with both EMRE and MCU via electrostatic interactions in low $\mathrm{Ca}^{2+}$. In high $\mathrm{Ca}^{2+}$, the MCU-MICU1 interaction is disrupted so that $\mathrm{Ca}^{2+}$ can pass the pore, but the EMRE-MICU1 interaction remains intact to keep MICU1 associated within the uniporter complex. (B) Uniporter holocomplex structures in low (PDB: 6WDN)/high $\mathrm{Ca}^{2+}(6 \mathrm{WDO})$. These structures recapitulate the key propositions in the occlusion model that MICU1 blocks MCU in low $\mathrm{Ca}^{2+}$ (left) but swings away to open the pore in high $\mathrm{Ca}^{2+}$ (right). (C) The potentiation model. This model proposes that MICU1 does not block MCU, but upon $\mathrm{Ca}^{2+}$ binding, MICUs increase the open probability of MCU by 2-fold via EMRE-dependent allosteric mechanisms. 


\section{Figure 2}

A

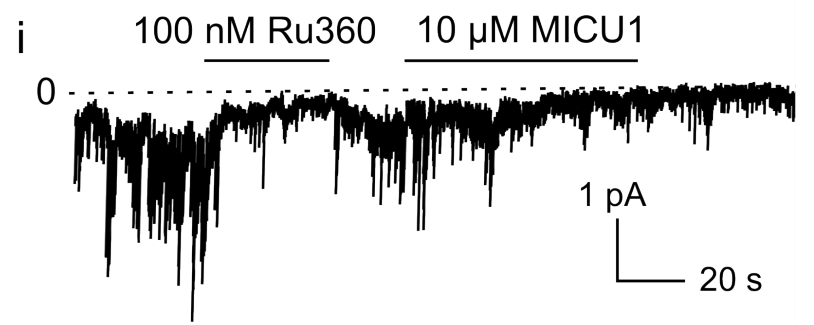

ii

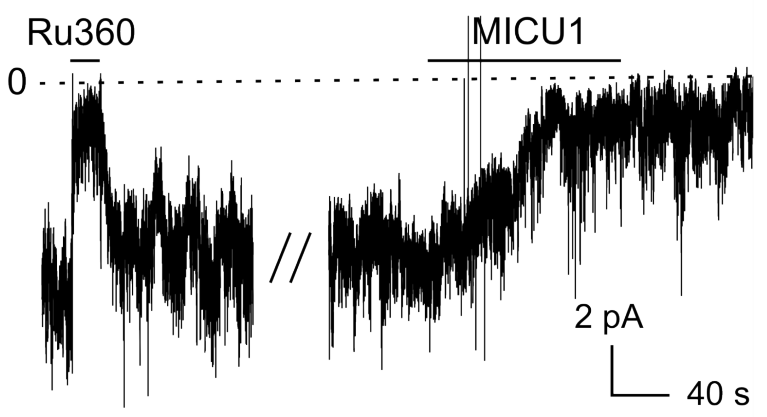

iii
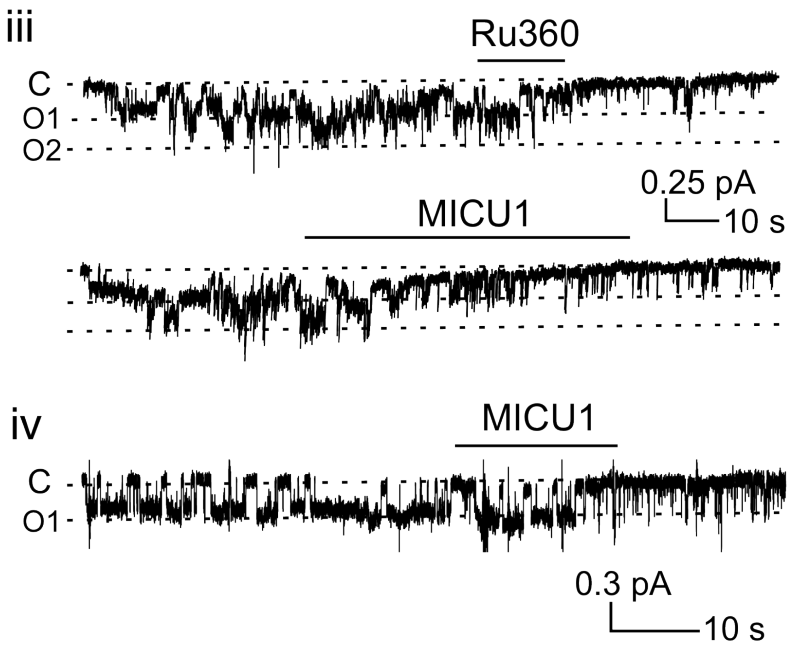

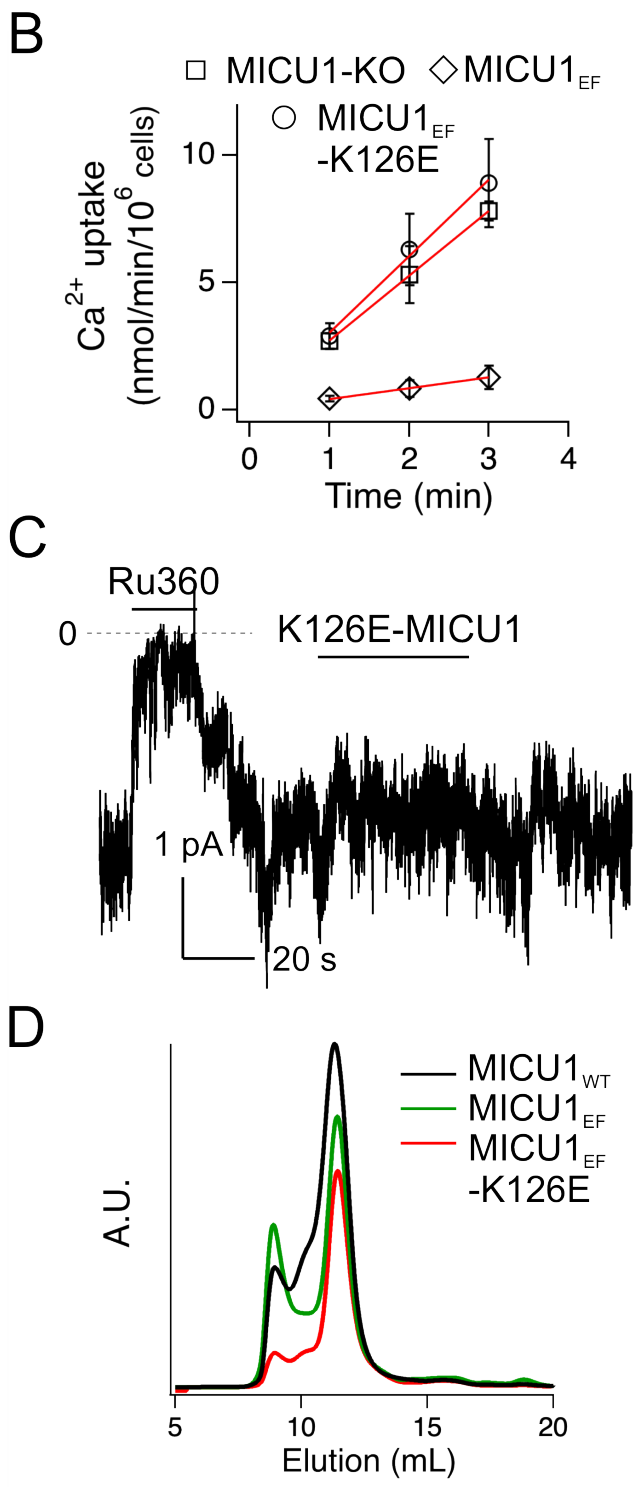

Figure 2. The effect of MICU1 $1_{\mathrm{EF}}$ on uniporter $\mathrm{Ca}^{2+}$ currents. (A) The effects of perfusing MICU1 $1_{\mathrm{EF}}$ to patches containing $\mathrm{Ca}^{2+}$-conducting hME. Membrane potentials were clamped at -60 to $-100 \mathrm{mV}$. MICU1 $1_{\mathrm{EF}}$ was tagged with an N-terminal maltose binding protein to facilitate proper protein folding in the periplasmic space of E. coli. In all recordings, [MICU1] and [Ru360] are $10 \mu \mathrm{M}$ and $100 \mathrm{nM}$, respectively. $\mathrm{N}=7$ (4 macroscopic recordings and 3 recordings with $1-2$ channels). (B) Mitochondrial uptake of ${ }^{45} \mathrm{Ca}^{2+}$ in MICU1-KO cells. The experiments were performed in the presence of $250 \mathrm{nM} \mathrm{Ca}^{2+}$, with MICU1-KO transiently expressing indicated MICU1 mutants. $\mathrm{N}=5$ (C) Mutational effects of $\mathrm{K} 126 \mathrm{E}$ on MICU1 function. Voltage was clamped at $-80 \mathrm{mV}$. N = 3. (D) Size exclusion profiles of WT MICU1 and MICU1 mutants. $\mathrm{N}=4-8$. 


\section{Figure 3}

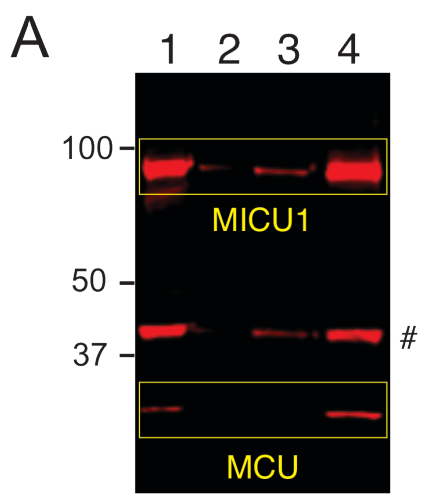

1. Mitochondria

2. Osmotic shock buffer

3. High salt buffer

4. Mitoplasts

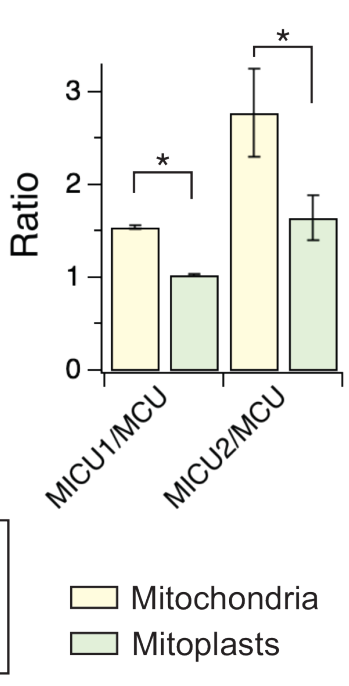

C

\section{Mitochondria}

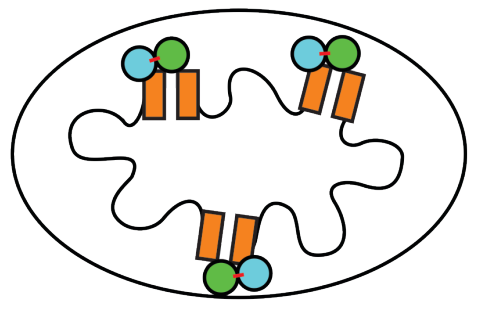

Mitochondria

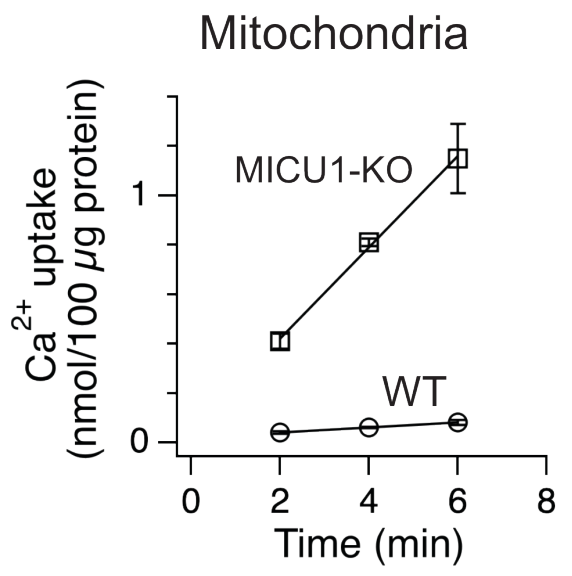

$\mathrm{D}$

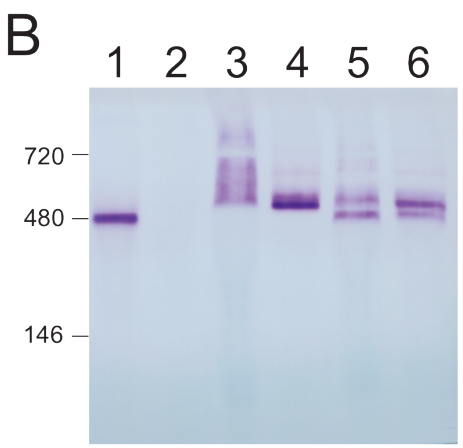

1. EMRE-KO mitoplasts

2. MCU/EMRE-KO mitoplasts

3. WT mitochondria

4. WT mitoplasts

5. MICU1-KO mitochondria

6. MICU1-KO mitoplasts

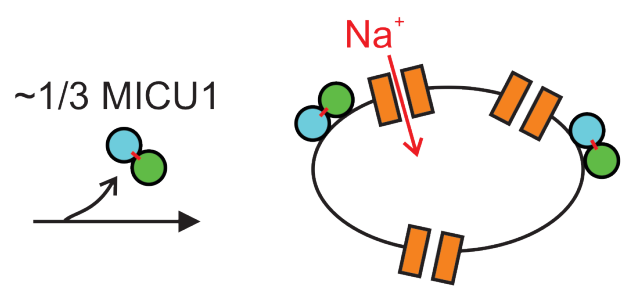

Mitoplasts

Mitoplasts

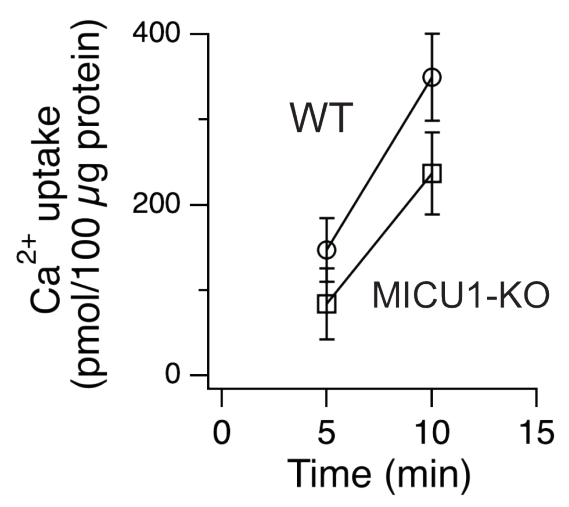

Figure 3. Biochemical and functional characterization of the uniporter in mitoplasts. (A) Changes of relative MICU/MCU levels during mitoplast preparation. The \# sign marks a non-specific band. $\mathrm{N}=4-$ 6. (B) BN-PAGE analysis of the uniporter complex. $\mathrm{N}=4$. (C) A cartoon explaining why MICU1 block is not observed in mitoplasts. The connected green-cyan balls represent the MICU1-MICU2 heterodimer connected by an intersubunit disulfide. (D) Deregulation of the uniporter in mitoplasts. The experiments were performed in the presence or absence of Ru360 to allow the calculation of uniporter-mediated, Ru360-sensitive $\mathrm{Ca}^{2+}$ uptake in mitochondria or mitoplasts. $\mathrm{N}=4-6$. 